\title{
Suppression of interferon-mediated anti-HBV response by single CpG methylation in the $5^{\prime}$-UTR of TRIM22
}

\author{
Keo-Heun Lim, ${ }^{1}$ Eun-Sook Park, ${ }^{1}$ Doo Hyun Kim, ${ }^{1}$ Kyung Cho Cho, ${ }^{2}$ Kwang Pyo Kim, ${ }^{2}$ \\ Yong Kwang Park, ${ }^{1}$ Sung Hyun Ahn, ${ }^{1}$ Seung Hwa Park, ${ }^{3}$ Kee-Hwan Kim, ${ }^{4}$ \\ Chang Wook Kim, ${ }^{5}$ Hong Seok Kang, ${ }^{1}$ Ah Ram Lee, ${ }^{1}$ Soree Park, ${ }^{1}$ Heewoo Sim, \\ Juhee Won, ${ }^{1}$ Kieun Seok, ${ }^{1}$ Jueng Soo You, ${ }^{6}$ Jeong-Hoon Lee, ${ }^{7}$ Nam-Joon $\mathrm{Yi}_{1}{ }^{8}$ \\ Kwang-Woong Lee, ${ }^{8}$ Kyung-Suk Suh, ${ }^{8}$ Baik L Seong, ${ }^{9}$ Kyun-Hwan Kim ${ }^{1,10,11}$
}

- Additional material is published online only. To view please visit the journal online (http://dx.doi.org/10.1136/ gutjnl-2016-312742).

For numbered affiliations see end of article.

\section{Correspondence to}

Professor Kyun-Hwan Kim, Department of Pharmacology, School of Medicine, Konkuk University, Seoul 05029, Korea; khkim10@kku.ac.kr.Tel

K-HL and E-SP contributed equally.

Received 30 July 2016 Revised 17 February 2017 Accepted 21 February 2017 Published Online First 23 March 2017

\section{Sinked}

- http://dx.doi.org/10.1136/ gutjnl-2017-314013

CrossMark

To cite: Lim K-H, Park E-S, Kim DH, et al. Gut 2017;67:166-178.

\section{ABSTRACT}

Objective Interferons (IFNs) mediate direct antiviral activity. They play a crucial role in the early host immune response against viral infections. However, IFN therapy for HBV infection is less effective than for other viral infections.

Design We explored the cellular targets of HBV in response to IFNs using proteome-wide screening. Results Using LC-MS/MS, we identified proteins downregulated and upregulated by IFN treatment in HBV $X$ protein $(\mathrm{HBX})$-stable and control cells. We found several IFN-stimulated genes downregulated by $\mathrm{HBx}$, including TRIM22, which is known as an antiretroviral protein. We demonstrated that HBx suppresses the transcription of TRIM22 through a single CpG methylation in its $5^{\prime}$-UTR, which further reduces the IFN regulatory factor-1 binding affinity, thereby suppressing the IFN-stimulated induction of TRIM22.

Conclusions We verified our findings using a mouse model, primary human hepatocytes and human liver tissues. Our data elucidate a mechanism by which HBV evades the host innate immune system.

\section{INTRODUCTION}

The battle between viral infection and the host defence system determines pathogenesis or cure of disease. During viral infections, hosts recognise viruses and effectively eradicate them through innate and adaptive immunity. However, viruses have various strategies to counteract and evade host immune systems.

At early stages of infection, interferons (IFNs) are the major effector cytokines against various pathogens during innate immune response. All types of IFNs (type I: IFN $\alpha$, IFN $\beta$ and IFN $\omega$; type II: IFN $\gamma$; type III: IFN $\lambda 1$, IFN $\lambda 2$ and IFN $\lambda 3$ ) are induced mainly through pathogen-associated molecules activated by pattern recognition receptors. ${ }^{2}$ IFNs secreted in response to virus infection ultimately induce multiple interferon-stimulated genes (ISGs), which have multiple antiviral and immunemodulating activities via IFNs' signalling pathways. ${ }^{3}$ Therefore, IFNs and their signalling pathways are targeted by viruses, which allow them to escape host immunity. ${ }^{1}$

\section{Significance of this study}

What is already known on this subject?

- Interferons (IFNs) mediate direct antiviral activity and play a crucial role in the host immune response against viral infections.

- IFN therapy for HBV infection is less effective than for other viral infections, implying that HBV has mechanisms to evade or counteract the IFN-mediated antiviral effects. However, the precise mechanism by which HBV escapes from IFNs remains largely unclear.

- TRIM22 is an IFN-stimulated gene and known as an antiviral protein against retroviruses, such as influenza virus, HIV and HBV.

What are the new findings?

- Proteome-wide analysis identifies HBV X protein ( $\mathrm{HBx}$ )-mediated desensitised proteins by IFN induction.

- HBV (via its protein $\mathrm{HBx}$ ) suppresses the transcription of TRIM22 through a single CpG methylation in its $5^{\prime}$-UTR, which reduces the IFN regulatory factor-1 binding, thereby suppressing the IFN-stimulated induction of TRIM22.

- Suppression of TRIM22 by HBx is involved in viral escape from IFN-mediated antiviral response.

- HBV suppresses TRIM22 expression in a mouse model, primary human hepatocytes and human liver tissues.

How might it impact on clinical practice in the foreseeable future?

- Our findings provide new insights that might be useful for counteracting the anti-IFN strategies of the virus and a potential way to improve the therapeutic effect of IFNs for HBV clearance.

- Our data elucidate the mechanism by which HBV evades the host innate immune system, and may provide a new and attractive therapeutic strategy for the cure of HBV infection. 
HBV infection causes chronic hepatitis, liver cirrhosis and even hepatocellular carcinoma. It is a major health problem, with over 350 million carriers worldwide. ${ }^{4} \mathrm{HBV}$, a noncytopathic hepatotropic DNA virus, has a distinct replication strategy through reverse transcription using pregenomic RNA (pgRNA) as a template in capsids. ${ }^{5}$ The synthesis of pgRNA and other viral RNAs is regulated by viral enhancers and core promoters. ${ }^{6}$ The HBV $\mathrm{X}$ protein $(\mathrm{HBx})$ is multifunctional, and its role is related to dysregulating cellular functions and to pathogenesis. $^{7}$ Notably, recent studies have revealed that HBx epigenetically regulates host proteins associated with $\mathrm{HBV}$-mediated liver pathogenesis. $^{89}$

Extensive studies have shown that IFNs suppress HBV replication. IFN $\alpha$ inhibits HBV replication through epigenetic regulation of $\operatorname{cccDNA}^{10}$ and MyD88-mediated pgRNA degradation. ${ }^{11}$ IFN $\gamma$ mainly mediates the antiviral effect of HBV-specific cytotoxic T lymphocytes. ${ }^{12}$ Both type I and type II IFNs inhibit HBV replication by disturbing the formation of replicationcompetent nucleocapsids containing $\operatorname{pgRNA}^{13}$ and accelerating their decay. ${ }^{14} \mathrm{HBV}$ replication is also restricted by the antiviral function of several ISGs such as MxA and APOBEC3G. ${ }^{15} 16$ Therefore, pegylated-IFN $\alpha$ is included in the current antiviral therapy regimen against $\mathrm{HBV}$ infection. ${ }^{17}$

However, only $30 \%-44 \%$ of patients with chronic hepatitis B show satisfactory virological and serological responses to treatment with pegylated-IFN $\alpha .{ }^{18}{ }^{19}$ Furthermore, the susceptibility of HBV to IFNs is lower than that of $\mathrm{HCV}^{20}$ These studies strongly imply that HBV has mechanisms to evade or counteract the IFN-mediated antiviral effects, which increase virus survival and cause resistance to IFN therapy. Some lines of evidence have shown that viral polymerase and $\mathrm{HBx}$ proteins are involved. ${ }^{21-23}$ However, the precise mechanism by which HBV escapes from IFNs remains largely unclear.

Proteins of the tripartite motif (TRIM) family are involved in various biological processes including oncogenesis, apoptosis and antiviral immune response. In particular, many members of the TRIM family induced in response to IFNs are associated with innate immunity and antiviral defence. ${ }^{24}$ Among them, TRIM22, one of the closest paralogs of TRIM5 $\alpha$, displays antiretroviral activity similar to that of TRIM $5 \alpha{ }^{25}$ Several studies have found that TRIM22 inhibits the transcription and replication of HIV through suppression of its long terminal repeat and disruption of the intracellular trafficking of the viral Gag protein. ${ }^{26} 27$ TRIM22 also restricts influenza virus and encephalomyocarditis virus by degrading a nucleoprotein and viral 3C protease, respectively, through its E3 ligase activity, ${ }^{28} 29$ suggesting the antiviral role of TRIM22 in a wide range of viral infections. Most importantly, TRIM22 has anti-HBV activity because it suppresses the HBV core promoter. ${ }^{30}$ Genome-wide microarray analysis revealed that viral clearance in HBVinfected chimpanzees is associated with TRIM22. ${ }^{31}$ Although the antiviral functions of TRIM22 have been extensively studied, the mechanism of viral evasion from TRIM22 is not yet known.

In this study, we demonstrate that HBV downregulates TRIM22 via epigenetic control, which makes it possible for the virus to evade the antiviral effect of IFNs during innate immune response. Our study provides new insights that might be useful for counteracting the anti-IFN strategies of the virus and also provides a foundation for improving the outcome of IFN therapy in patients with HBV infection. Our data also explain why coinfection with HBV and HIV has a worse prognosis than infection with HBV alone.
RESULTS

Proteome-wide screening for HBx-mediated desensitised proteins by IFN treatment: identification of TRIM22 as a possible target for immune evasion

Response to IFNs and their subsequent antiviral activity that induces ISGs can be impaired by a variety of viruses, protecting them from IFNs. ${ }^{1}$ In HBV, the HBx protein is a key player regulating the expression of host proteins by epigenetic modification. $^{8} 9$ In this regard, we investigated the possible targets involved in HBV protection from IFNs. First, we performed proteome-wide analysis using LC-MS/MS after treatment of $\mathrm{HBx}$-expressing and control (transfected with pcDNA) cells with or without IFN $\gamma$ (figure 1A).

A total of 1406 proteins were identified from four different groups (IFN $\gamma-/ \mathrm{pcDNA}, \mathrm{IFN} \gamma-/ \mathrm{HBx}, \mathrm{IFN} \gamma+/ \mathrm{pcDNA}$ and IFN $\gamma$ $+/ \mathrm{HBx}$ cells), each of which included 855, 946, 832 and 822 proteins, respectively. They were identified using LC-MS/MS analysis following FASP digestion and off-gel fractionation (see online supplementary information for details). Within each group, proteins identified only in one other group were assigned to Cluster A, B, C or D (figure 1B). According to this classification, 110 proteins in Cluster $\mathrm{D}$ were upregulated by both IFN $\gamma$ and $\mathrm{HBx}$, and 166 proteins in Cluster $\mathrm{C}$ were upregulated by IFN $\gamma$ (IFN-stimulated proteins, ISPs), but were suppressed by HBx.

All identified proteins were classified by Gene Ontology using DAVID bioinformatics resources (http://david.abcc.ncifcrf.gov). Among the putative biological functions, we focused on two categories, 'Immune Response' and 'Response to Virus'. Differential expression of proteins in each category is shown in figure $1 \mathrm{C}$. The information about eight ISPs from Cluster $\mathrm{C}$ suppressed by $\mathrm{HBx}$ is summarised in online supplementary table S1. Notably, only TRIM22 and TWF2 were detected in both categories (Immune Response and Response to Virus). Since TRIM22 was reported to have antiviral activity against several viruses including $\mathrm{HBV}^{25}$ we focused on TRIM22 as a potential target of HBV. Western blot analysis confirmed that TRIM22 is induced by IFN $\gamma$; however, this effect was considerably desensitised by $\mathrm{HBx}$ (figure 1D).

\section{HBV desensitises the expression of IFN-induced TRIM22 through the viral protein $\mathrm{HBx}$}

To investigate whether $\mathrm{HBV}$ and $\mathrm{HBx}$ regulate the expression of TRIM22, we first examined the basal levels of TRIM22 mRNA after transfection with replication-competent WT HBV1.2mer (WT HBV1.2), HBx-deficient HBV genome (HBV1.2(X-)) or pcDNA-HBx-HA. Both WT HBV1.2 and HBx-HA decreased the expression of TRIM22 in a dose-dependent manner, whereas HBV1.2(X-) did not significantly change the level of TRIM22 mRNA (figure 2A and see online supplementary figure $\mathrm{S} 1 \mathrm{~A})$. Furthermore, HBx suppressed the induction of TRIM22 upon IFN $\gamma$ treatment (figure $2 \mathrm{~B}$ and see online supplementary figure S1B). Among IFNs, IFN $\gamma$ strongly induced TRIM22 expression, whereas IFN $\beta$ showed little effect (figure 2C and see online supplementary figure S2). The activities of IFNs were verified by the induction of ISGs including TRIM22 (see online supplementary figure S2).

The suppression of TRIM22 by HBx was further confirmed by western blot analysis using the virus and $\mathrm{HBx}$-encoding plasmid (figure 2D). TRIM22 expression was significantly suppressed by HBx (figure 2E). The time course of TRIM22 induction by IFN $\gamma$ was considerably disrupted in $\mathrm{HBx}$-expressing cells (figure $2 \mathrm{~F}$ and see online supplementary figure S1B). In 
A

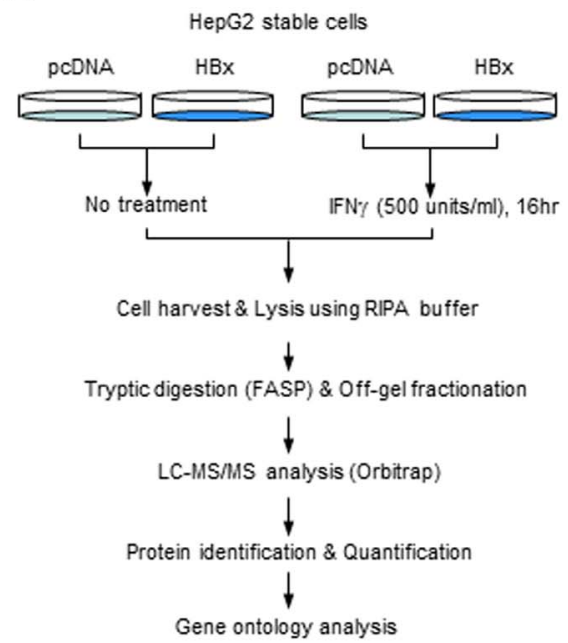

C

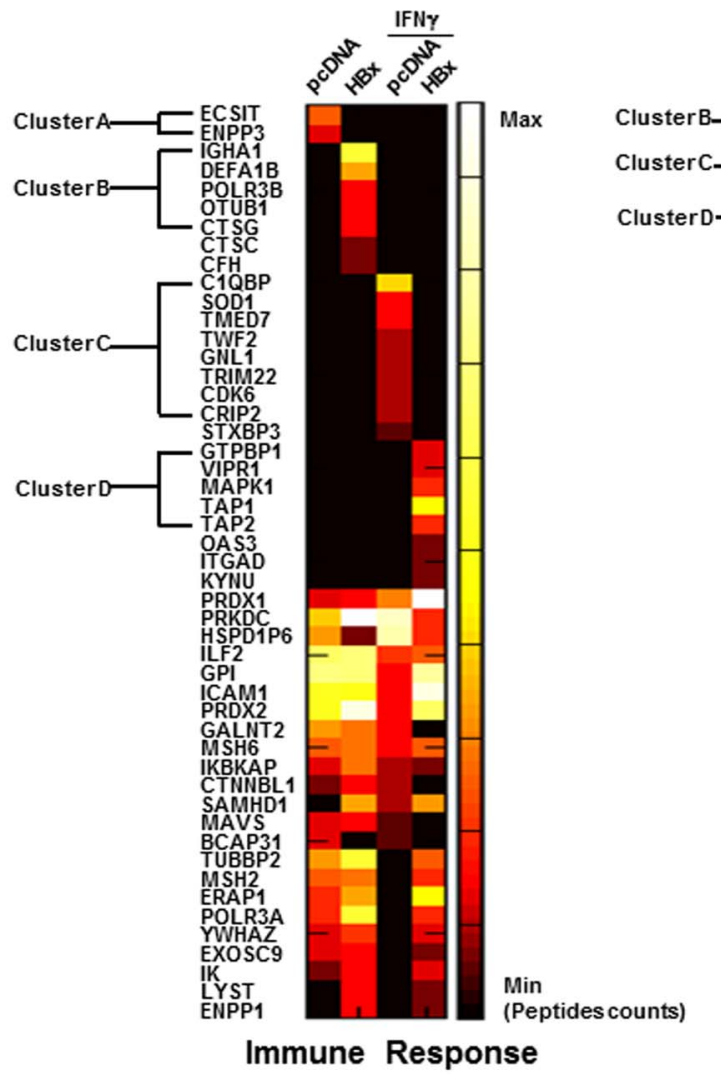

B

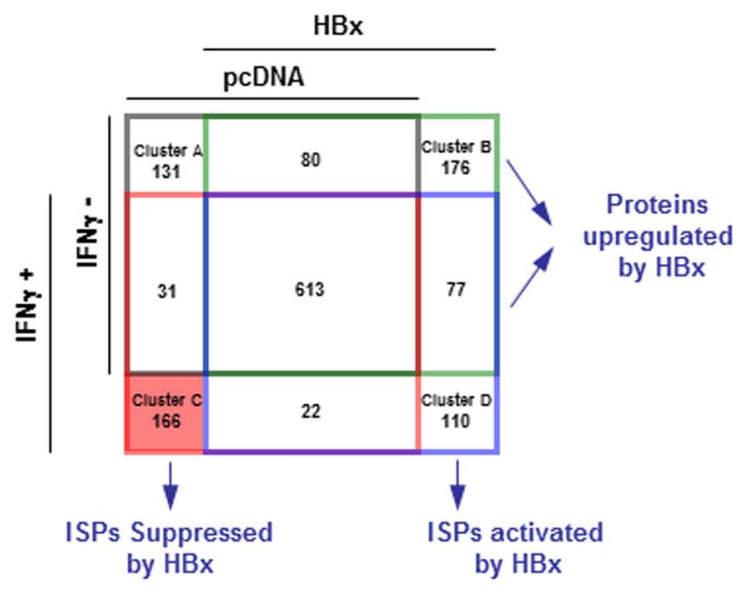

addition, only HBx suppressed the induction of TRIM22 by IFN $\gamma$, whereas other viral proteins including polymerase and core proteins had no effect in either HepG2-NTCP or HepaRG infection systems (figure $2 G$ ).
Since the anti-HBV effect of TRIM22 occurs in the nucleus through inhibition of the core promoter, ${ }^{30}$ we checked the nuclear level of TRIM22. As shown in online supplementary figure S3, the proportion of TRIM22 in the 
A

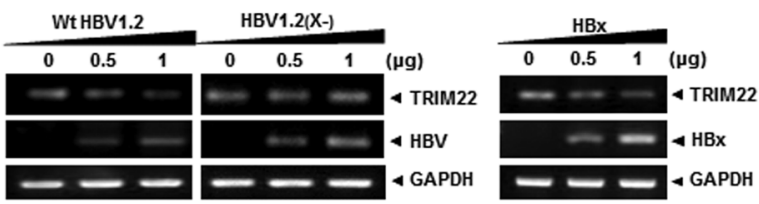

B

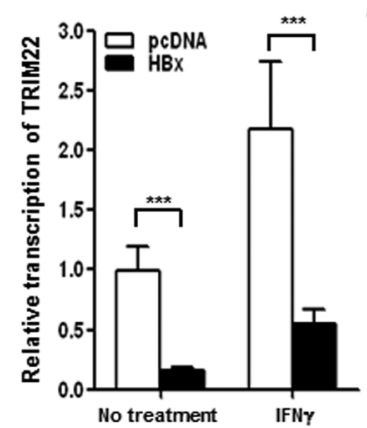

F
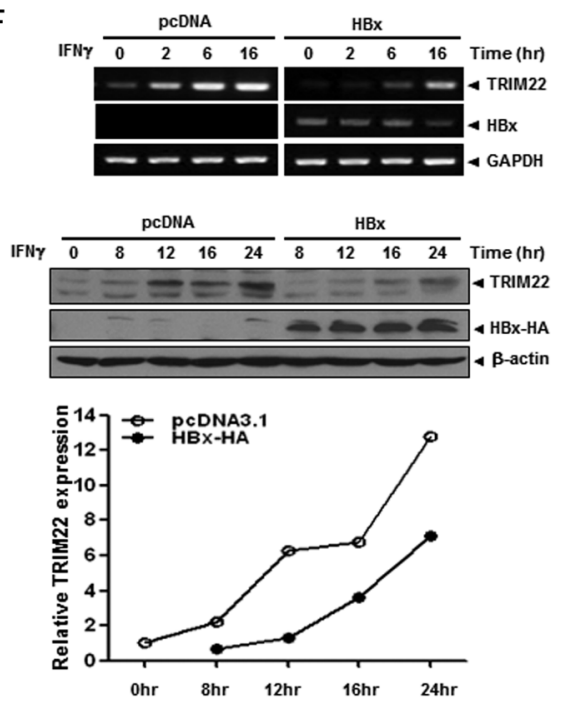

E

G
D

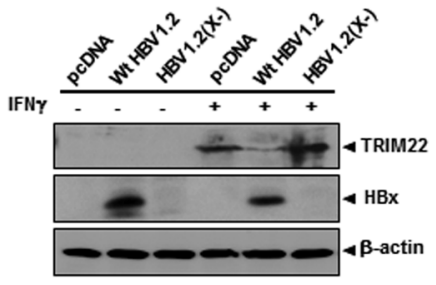

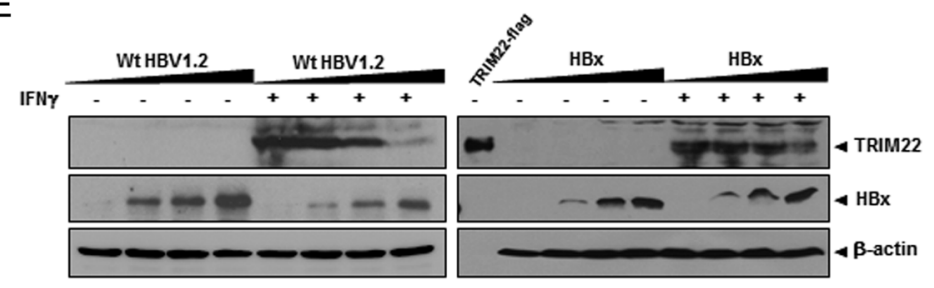

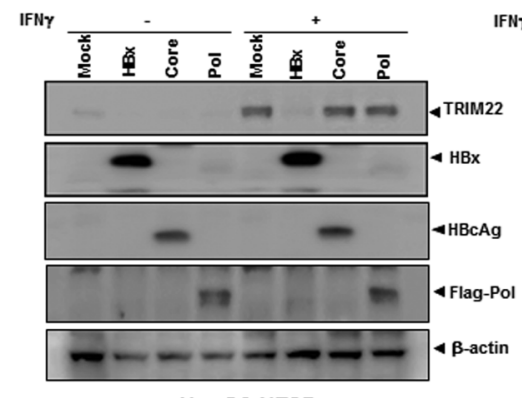

HepG2-NTCP

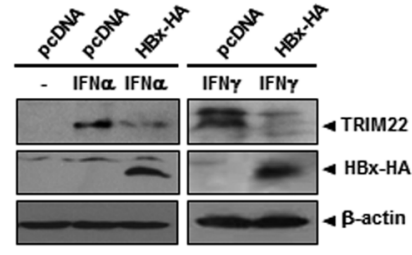

Figure 2 HBV suppresses the expression of TRIM22 induced by interferons (IFNs) through the viral protein HBV X protein (HBX). (A) Inhibition of TRIM22 transcription by HBx. Huh7 cells were transfected with the indicated plasmids. After 48 hours, cells were harvested and analysed by semiquantitative RT-PCR. (B) Effect of HBx on the level of TRIM22 mRNA in cells treated with or without IFN $\gamma(2 \mathrm{hours}, 500 \mathrm{units} / \mathrm{mL})$. Data are from three independent real-time PCR analyses $\left({ }^{* * *} \mathrm{p}<0.001\right)$ and normalised to the expression level of GAPDH. (C) Effect of IFNs on TRIM22 expression. HepG2 cells were treated with the indicated IFNs ( 500 units $/ \mathrm{mL}$ ) for 24 hours and analysed by western blotting. (D) HBx-mediated suppression TRIM22 expression induced by IFN $\gamma$. Cells transfected with $2 \mu \mathrm{g}$ of indicated plasmids were treated with IFNs ( $500 \mathrm{units} / \mathrm{mL}$ ) for 24 hours, and the expression level of TRIM22 was analysed by western blotting. (E) Dose-dependent suppression of IFN-inducible TRIM22 by HBx. HepG2 cells were transfected with increasing amounts $(0,1,2$ and $4 \mu \mathrm{g})$ of the indicated plasmids and treated with IFN $\gamma$ for 24 hours. Total DNA amounts were adjusted to $4 \mu \mathrm{g}$ using pcDNA3.1. The lysate of cells overexpressing TRIM22-flag was used as a positive control. (F) Effect of HBx on the expression kinetics of TRIM22 in the presence of IFN $\gamma$. HepG2 cells were transfected with HBx-HA (2 $\mu$ g) and treated with IFN $\gamma$ for indicated time. The levels of TRIM22 mRNA and protein were analysed by semiquantitative RT-PCR and western blotting, respectively. The relative expression of TRIM22 protein was measured by GelQuant.NET analysis software (Biochem Lab Solutions). (G) Effect of viral proteins on TRIM22 expression in HepG2-NTCP and HepaRG cells. Cells transfected with $2 \mu \mathrm{g}$ of indicated plasmids were treated with IFNs (500 units/mL) for 24 hours, and the expression levels of TRIM22 and viral proteins were analysed by western blotting.

nucleus after IFN $\gamma$ treatment was not altered by the presence of HBx.

\section{Identification of the element responsible for HBx-mediated suppression of TRIM22}

To identify the elements responsible for HBx-mediated suppression of TRIM22, we constructed seven luciferase reporter plasmids, including five serial deletion constructs spanning $2.5 \mathrm{~kb}$ upstream of the transcription start site of the TRIM22 gene. Construct TP6 contained 5'-UTR, and construct TP7 contained both the $5^{\prime}$-UTR and the p53 response element in intron 1 , which was suggested to be necessary for promoter activity ${ }^{32}$ (figure 3A). The luciferase assay revealed that TP6 and TP7 have strong basal promoter activity, suggesting that the $5^{\prime}$-UTR $(+1$ to +211$)$ is essential for TRIM22 transcription (figure 3A). Further analysis demonstrated that the $5^{\prime}$-UTR is also responsible for both TRIM22 induction (figure $3 \mathrm{~B}$ ) and $\mathrm{HBx}$-mediated desensitisation of TRIM22 induction by IFN $\gamma$ (figure 3C). Interestingly, this region resembles a IFN $\gamma$-stimulating element, a region that binds IFN regulatory factor-1 (IRF1). ${ }^{33}$ Accordingly, 
we examined whether the HBx-mediated suppression of TRIM22 is regulated by IRF1. IFN $\gamma$ but not $\mathrm{HBV}$ or $\mathrm{HBx}$ increased IRF1 expression (see online supplementary figure S4). These findings suggest that the $5^{\prime}$-UTR $(+1$ to +211$)$ is critical for transcriptional activity, induction by IFNs and $\mathrm{HBx}^{-}$ mediated suppression of TRIM22 expression.
HBx represses IFN-induced TRIM22 expression through epigenetic regulation

Previously, we and other groups reported that $\mathrm{HBx}$ regulates the expression of several host proteins through epigenetic control, mainly DNA methylation in their regulatory regions. ${ }^{8} 9$ Therefore, we examined whether downregulation of TRIM22
A

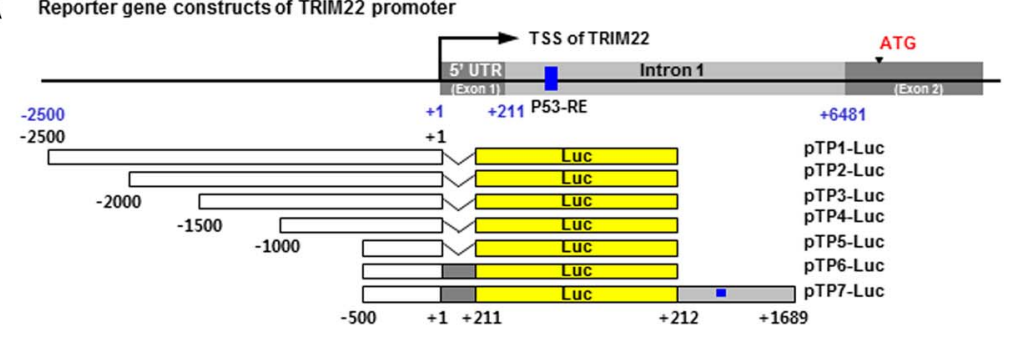

B
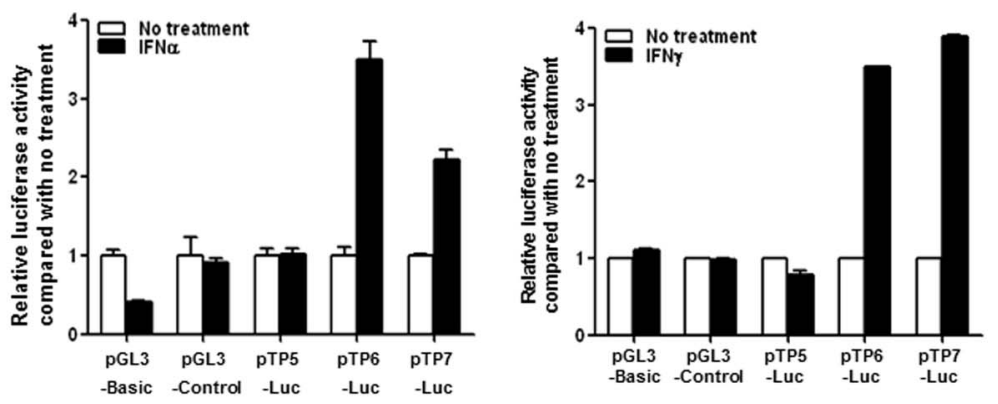

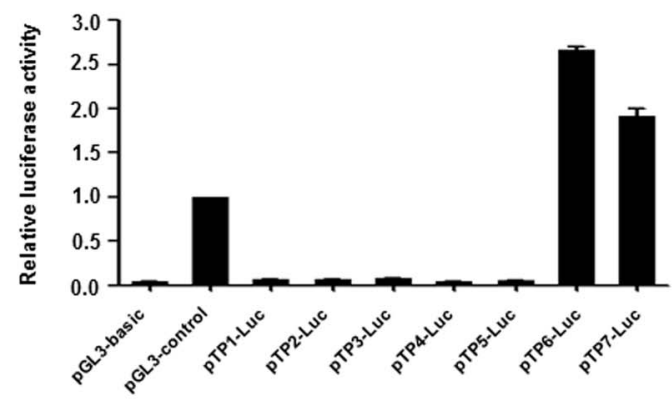

C

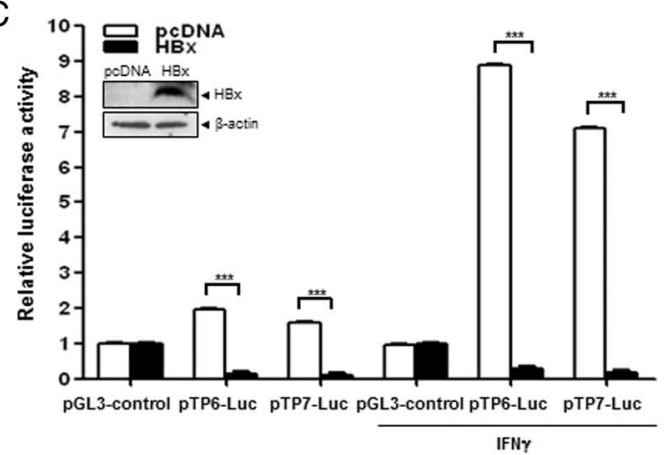

D

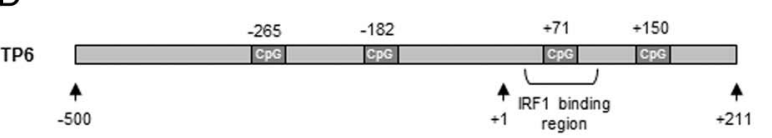

E
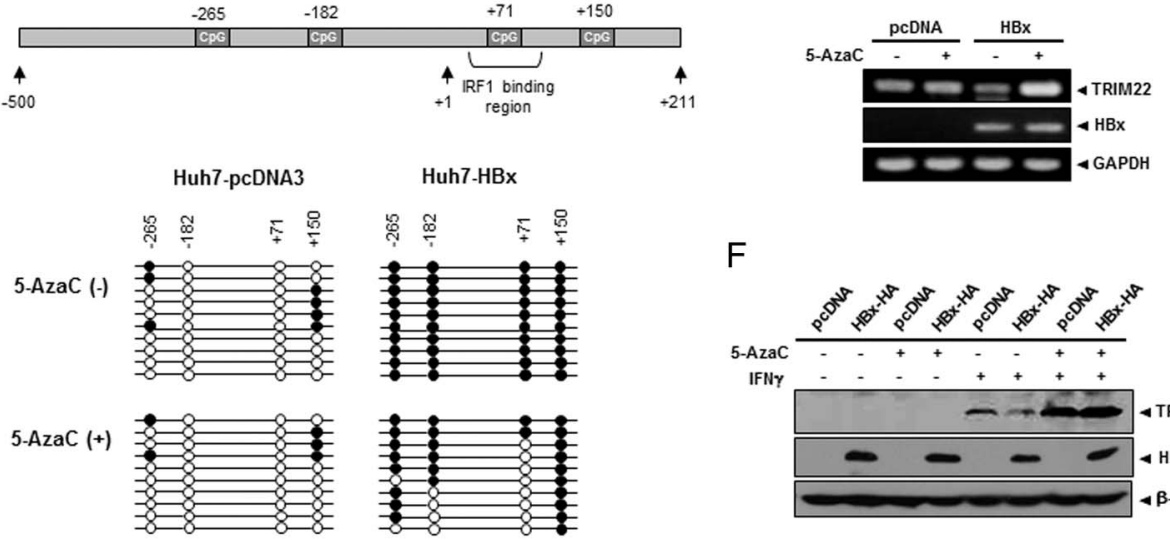

F

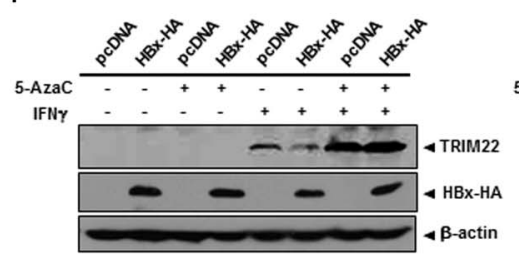

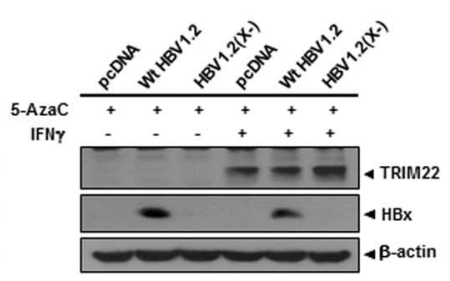

Figure 3 HBV X protein (HBX) suppresses TRIM22 induced by interferons (IFNs) through epigenetic regulation in the $5^{\prime}$-UTR of TRIM22. (A) Schematic representation of the promoter and 5'-UTR of TRIM22. Seven luciferase reporter plasmids (pTP1-Luc to pTP7-Luc) that included the putative TRIM22 promoter were constructed. Basal activity of putative TRIM22 promoters was determined by luciferase reporter assay. pGL3-basic and pGL3-control (SV40 promoter) plasmids were used as negative and positive controls, respectively. TSS, transcription start site; UTR, untranslated region; p53-RE, p53 response element. (B) Effect of IFNs on the induction of TRIM22 promoter. HepG2 (left panel) and Huh7 (right panel) cells were transfected with reporter plasmids $(0.25 \mu \mathrm{g})$ and treated with or without IFN $\alpha$ or IFN $\gamma(500$ units $/ \mathrm{mL})$ for 24 hours, and luciferase activity was determined. (C) HBx suppresses the expression of TRIM22 regardless of IFN treatment. At 24 hours post transfection with reporter plasmids, cells were treated with IFN $\gamma(500$ units $/ \mathrm{mL}$ ) for 24 hours, and luciferase activity was measured. The expression of HBx was confirmed by western blotting. Data are mean $\pm S D$ of at least three independent experiments; ${ }^{* * *} p<0.001$. (D) Upper panel, a schematic outline of the four single CpG sites in the promoter and 5'-UTR of TRIM22. The third CpG belongs to the IFN regulatory factor-1 (IRF1)-binding region. Lower panel, effect of HBx on CpG methylation. The results of bisulfite sequencing with or without $5 \mathrm{AzaC}$ treatment are shown. The methylation status of 10 clones from each sample was analysed. Open circles, CpG; filled circles, methyl-CpG. (E and F) Treatment with 5AzaC restores the HBx-mediated suppression of TRIM22. Cells were transfected with $2 \mu \mathrm{g}$ of the indicated plasmids and were treated with $5 \mathrm{AzaC}$ with or without IFN $\gamma$ treatment. The TRIM22 mRNA (E) and protein (F) levels were determined by semiquantitative RT-PCR and western blot analysis, respectively. $5 \mathrm{AzaC}(10 \mu \mathrm{M})$ was present throughout the cell culture. IFN $\gamma(500$ units/mL) was added for 24 hours before harvesting. 
by HBx is associated with epigenetic modification. We first analysed the CpG sites using TP6, which contains the $5^{\prime}$-UTR $(+1$ to +211$)$. There were only four CpGs scattered from upstream of $500 \mathrm{bp}$ to downstream of $200 \mathrm{bp}$ (figure 3D, upper panel). Interestingly, bisulfite sequencing revealed that each $\mathrm{CpG}$ site in the TRIM22 promoter was fully methylated in HBx-expressing cells, unlike in control cells (figure 3D, middle panel). Unexpectedly, the four CpG sites showed different sensitivity to 5'-aza-2'-deoxycytidine (5AzaC), a DNA methylation inhibitor (figure 3D, lower panel). Methylation at the first and last $\mathrm{CpG}$ sites was stable, but the third CpG site (+71) was unmethylated after $5 \mathrm{AzaC}$ treatment. This suggests that methylation of the third CpG site plays a critical role in the suppression of TRIM22 transcription.

Overexpression of the DNA methyltransferases DNMT1, DNMT3A1 or DNMT3A2 did not affect the expression level of TRIM22 (see online supplementary figure S5A). The expression levels of DNA methyltransferases were not considerably affected by $\mathrm{HBx}$ in HepG2 cells (see online supplementary figure S5B).

As shown in figure $3 \mathrm{E}, \mathrm{F}$ (left panel), the mRNA and protein levels of TRIM22, which had been dramatically repressed by $\mathrm{HBx}$, were restored by $5 \mathrm{AzaC}$ treatment. The repression of TRIM22 by genome-driven HBx (figure 2D) was also completely abolished by $5 \mathrm{AzaC}$ treatment (figure $3 \mathrm{~F}$, right panel). These results suggest that the suppression of TRIM 22 by HBx is mediated by epigenetic regulation of the TRIM22 promoter.

\section{Methylation of single $\mathrm{CpG}(+71)$ by $\mathrm{HBx}$ is responsible for HBx-mediated desensitisation of TRIM22 induction}

Correlation between hypomethylation of the third CpG $(+71)$ (figure 3D) and restoration of TRIM22 expression (figure 3E, F) by $5 \mathrm{AzaC}$ treatment elucidates the role of this site in IFN-mediated TRIM22 induction. We generated five TP6 mutant reporter clones containing one $\mathrm{CpG}$ mutation each (figure 4A) and examined their basal and IFN-induced promoter activities. The results of luciferase assay showed that all mutants except pTP6-M5 lost their induction activities; pTP6-M5 had substitutions in all CpGs except the third one and maintained its IFN $\alpha$ and IFN $\gamma$-induced activity (figure 4B). HBx strongly suppressed pTP6-M5 activity under both basal conditions and upon IFN $\gamma$ treatment (figure 4C). These results demonstrate that a single $\mathrm{CpG}(+71)$ is responsible for the suppression of TRIM22 transcription by HBx.

As mentioned before, CpG $(+71)$ is located in the IRF1-binding region (figure 4A). ${ }^{33}$ Therefore, we examined the possibility that methylation at this site impedes IRF1 binding, which is essential for IFN $\gamma$-induced TRIM22 expression. We performed electrophoretic mobility shift assay (EMSA) using four DNA probes with comparable radioactivity (figure 4D). Nuclear IRF1 was detected only in IFN $\gamma$-treated cells (figure 4E, bottom panel). IRF1 bound to the wild-type probe harbouring a single CpG (+71), as revealed by cold competition and antibody supershift assays (figure 4E, left panel). Both the AG and TG probes lost their ability to bind IRF1 dramatically (figure 4E), in line with abrogated induction activity of pTP6-M2 and pTP6-M3 (figure 4B). In EMSA using the methylated CG probe, the binding affinity of IRF1 was markedly lower than that of the wild-type probe, in which a strong band was observed (figure 4E, right panel). This result implies that IRF1 binding is impeded when the CpG (+71) site is methylated, and confirms the importance of this site in IRF1 binding and TRIM22 induction.
We further investigated the influence of $\mathrm{HBx}$ on $\mathrm{CpG}(+71)$. In chromatin immunoprecipitation assay, IFN $\gamma$-induced IRF1 interacted with the IRF1-binding site in control cells; this interaction was markedly diminished in HBx-expressing cells (figure 4F). IRF1 expression was not altered by HBx. Treatment with 5 AzaC completely restored IRF1 binding in HBx-expressing cells, suggesting that $\mathrm{CpG}$ methylation by $\mathrm{HBx}$ disturbs the IRF1-DNA interaction and downregulates IFN-induced TRIM22 expression.

\section{Suppression of TRIM22 by HBx is involved in viral escape from IFN-mediated antiviral response}

TRIM22 exhibits anti-HBV activity through transcriptional suppression of the HBV core promoter, ${ }^{30}$ whereas HBx epigenetically desensitises TRIM22 expression. We wondered how HBx affects the anti-HBV activity of IFNs. We chose IFN $\gamma$ to test its anti-HBV effect, since IFN $\gamma$ induces TRIM 22 more prominently than does IFN $\alpha$ (figure 2C) and efficiently inhibits viral replication and transcription. Southern and western blot analyses showed that the amounts of HBV DNA and proteins (HBs and core) were reduced in response to IFN $\gamma$ in a dose-dependent manner (figure 5A, left panel). The transcription of HBV genomic and subgenomic RNAs was also significantly reduced by IFN $\gamma$ (figure $5 \mathrm{~A}$, right panel). In contrast, the expression level of TRIM22 was inversely related to HBV replication (figure 5A, left panel), suggesting its role in IFN $\gamma$-mediated anti-HBV activity.

To examine whether IFN $\gamma$-induced anti-HBV activity is mediated by TRIM22, we analysed enhancer activity and the levels of HBV RNA, DNA and proteins after TRIM22 knockdown by siRNA. siTRIM22 effectively knocked down overexpressed TRIM22-flag as well as IFN-induced TRIM22; however, it did not affect the expression of HBx (see online supplementary figure S6). Since TRIM22 inhibits the activity of the HBV enhancer and core promoter, ${ }^{30}$ we constructed a reporter plasmid containing HBV Enhancer I/II and core promoter (pEnhI-II-Luc) to investigate the effect of TRIM22 on promoter activity (figure $5 \mathrm{~B}$, upper panel). Anti-HBV activities mediated by TRIM 22 overexpression or IFN $\gamma$ treatment were significantly reduced by siTRIM22 (figure $5 \mathrm{~B}$ ). HBV transcription and expression of viral core and surface proteins initially reduced by TRIM22 or IFN $\gamma$ were markedly restored by siTRIM22 (figure 5C, D, left panel). This result suggests that IFN $\gamma$-induced anti-HBV activity is mediated by TRIM22. In particular, the HBV genomic RNA (3.5 kb), a template for genomic DNA replication and mRNA for the core protein, was further recovered by TRIM22 knockdown (figure 5D, lower right panel). The levels of total viral and subgenomic $(2.4 / 2.1 \mathrm{~kb}$, mRNAs for surface proteins) RNAs were also significantly recovered (figure 5D, upper right panel). We further examined the effect of TRIM22 knockdown on IFN-mediated antiviral activity using the HepG2-NTCP and HepaRG HBV infection systems. Genome replication and the expression of viral proteins were almost completely restored by TRIM22 knockdown in both systems (figure 5E, F). Notably, the basal level of TRIM22 was much higher in HepaRG than in HepG2 cells (figure 5F). These results suggest an important role of TRIM22 under physiological conditions.

Finally, we compared IFN $\gamma$-induced anti-HBV activity with and without $5 \mathrm{AzaC}$ treatment to see if the HBV evasion mechanism against IFN $\gamma$ antiviral response relies on HBx-mediated epigenetic suppression of TRIM22. Viral RNAs and HBx initially inhibited by IFN $\gamma$ were further decreased by $5 \mathrm{AzaC}$ 
A

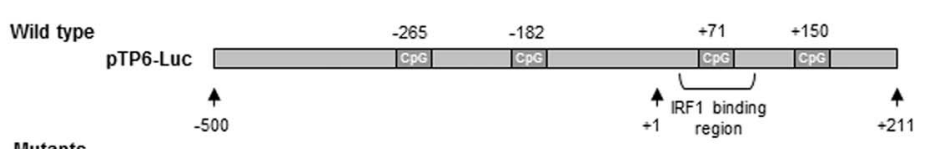

Mutants

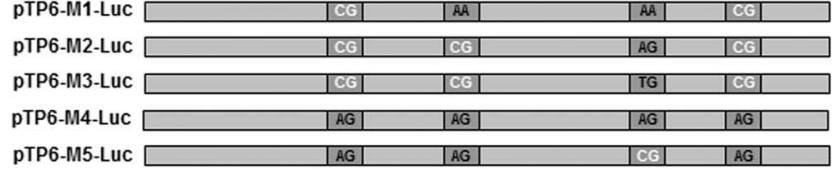

B
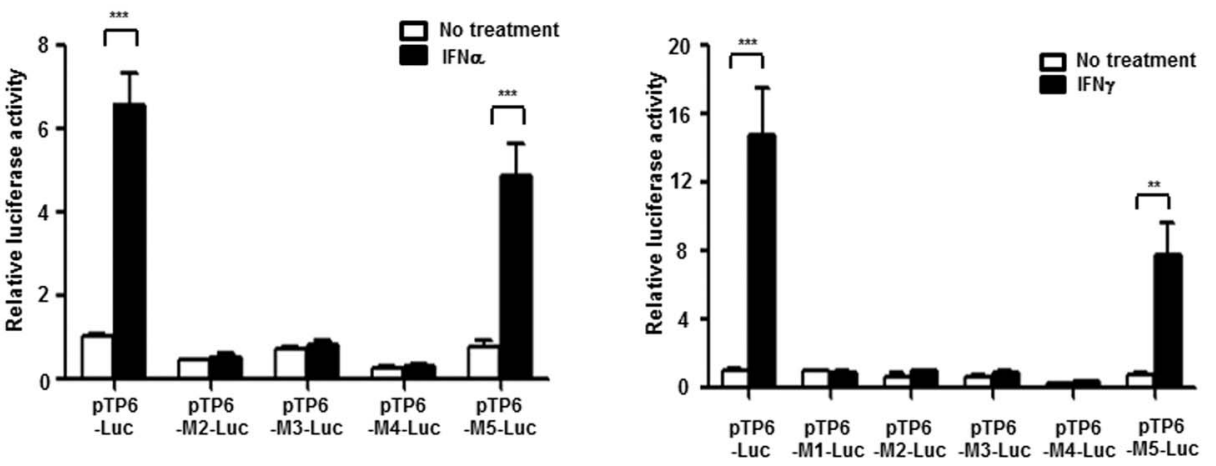

D

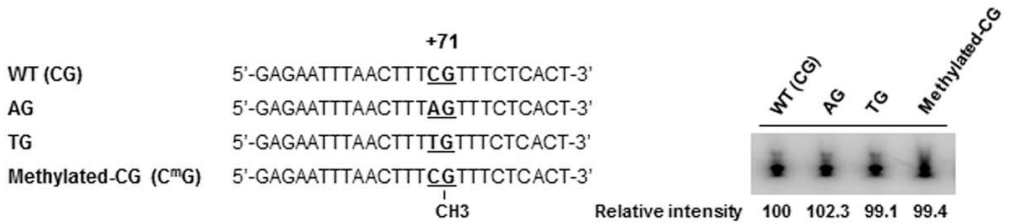

E

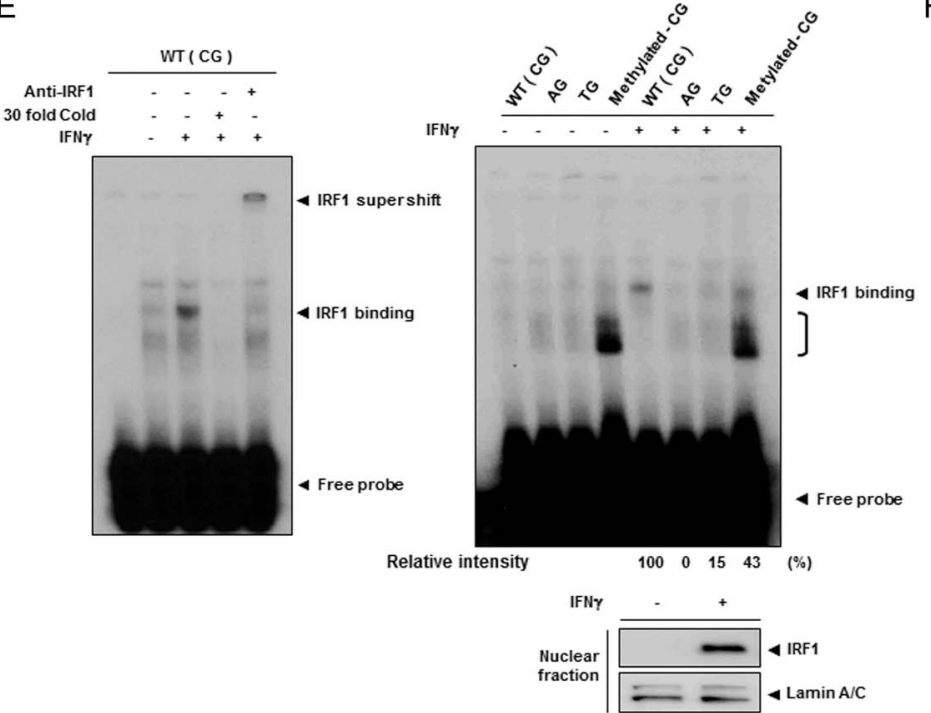

F

No treatment

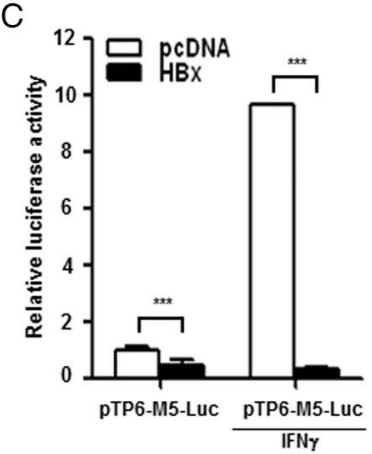

Figure 4 HBV X protein (HBx) suppresses TRIM22 expression through methylation of a single CpG in the 5'-UTR of TRIM22. (A) Schematic representation of five mutant luciferase reporter clones. Cytosines or guanines of $\mathrm{CpG}$ were mutated to adenine or thymine. (B) Induction of mutant promoters by interferons (IFNs). HepG2 cells were transfected with the indicated reporter plasmids $(0.5 \mu \mathrm{g})$, treated with or without IFN $\alpha$ or IFN $\gamma$ ( 500 units $/ \mathrm{mL}$ ) for 24 hours, and luciferase activity was measured. (C) Suppression of the M5 TRIM22 promoter by HBx regardless of IFN $\gamma$ treatment. Cells were transfected with pTP6-M5-Luc $(0.5 \mu \mathrm{g})$, and basal and IFN $\gamma$-induced promoter activity was measured. Data are mean \pm SD of at least three independent experiments; ${ }^{* *} p<0.001,{ }^{* *} p=0.003$. (D) Sequences of $\left[{ }^{32} \mathrm{P}\right]$-labeled probes used for IFN regulatory factor-1 (IRF1) electrophoretic mobility shift assay (EMSA). The single CpG site $(+71)$ is underlined, and mutations or methylated CpG are indicated. The radioactivity of each $\left[{ }^{32} \mathrm{P}\right]$-labeled probe was determined. (E) Verification of IRF1 interaction with WT probe through antibody supershift and competition assay with a cold probe (left panel). (E) EMSA of binding affinity between IFN-induced IRF1 and each probe. Induction of IRF1 by IFN $\gamma$ (500 units/mL) was confirmed by western blot analysis of nuclear extracts (lower panel). The bracket indicates the complex formed by methylated CpG-binding proteins. Relative intensity was calculated by subtracting the intensity without treatment from that with IFN $\gamma$ treatment (right panel). (F) Chromatin immunoprecipitation (ChIP) assay of the TRIM22 promoter region with IRF1 antibody and genomic DNA obtained from Huh7 cells stably expressing $\mathrm{HBx}$ and control cells. Both cell groups were treated with or without IFN $\gamma(500$ units $/ \mathrm{mL})$ or $5 \mathrm{AzaC}(10 \mu \mathrm{M})$, and ChIP assay was performed. Circles indicate single CpG sites. R2 was used as a negative control. Induction of IRF1 by IFN $\gamma$ was confirmed by western blotting (lower panel). 
A

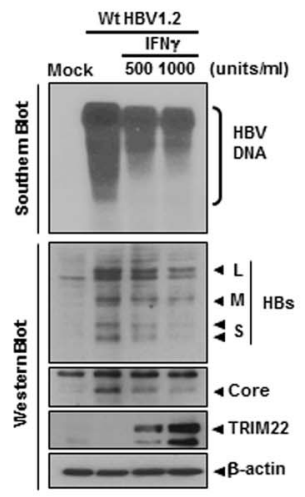

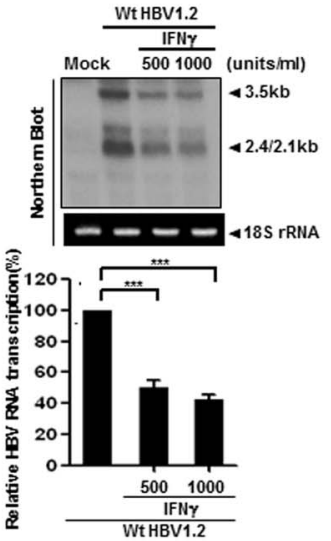

C

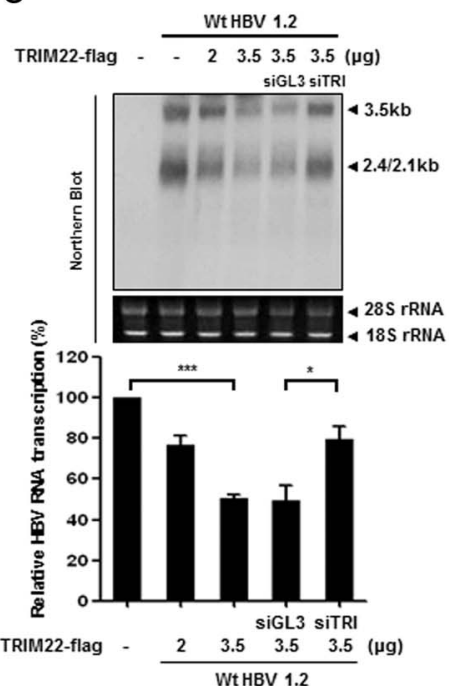

B

pEnhl-II-Luc construct
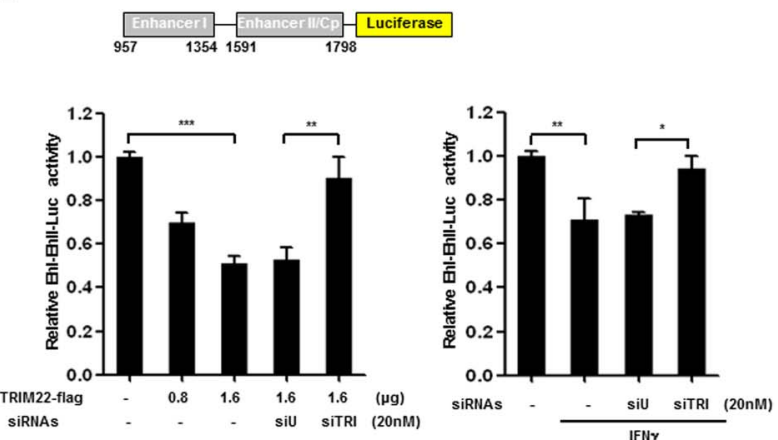

D
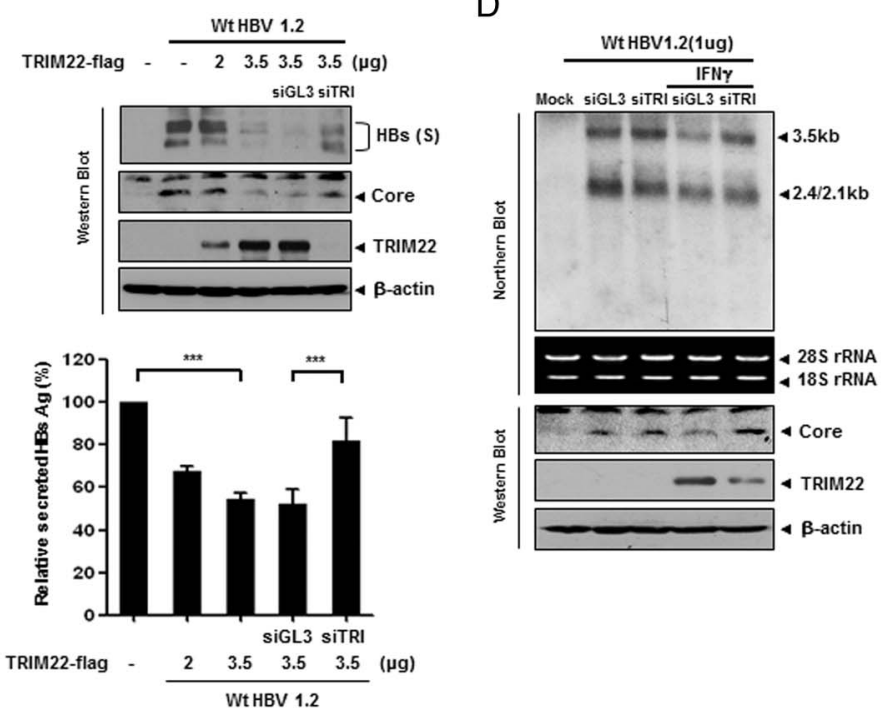

Figure 5 Suppression of TRIM22 by HBV X protein (HBx) is involved in HBV evasion from interferon (IFN)-mediated anti-HBV response. (A) IFN $\gamma$ inhibits HBV replication and transcription and involvement of TRIM22 induction. HepG2 cells were transfected with WT HBV1.2 $(1 \mu \mathrm{g})$ and treated with IFN $\gamma$ for 48 hours. Genome replication, expression of TRIM22 and viral surface/core proteins (left panel) and transcription of pregenomic RNA (pgRNA)/subgenomic RNAs (right panel) were analysed by southern, western and northern blotting, respectively. Relative level of HBV transcription was calculated using a phosphorimager; data are from at least three independent experiments $\left({ }^{* * *} p<0.001\right)$. (B) Left panel, effect of TRIM22 knockdown on Enhancer I/II activity. HepG2 cells were transfected with plasmids (pEnhl-II-Luc $(0.4 \mu \mathrm{g})$, pCD-TRIM22-flag and p $\beta$-gal $(0.25 \mu \mathrm{g})$ ) with or without indicated siRNAs $(20 \mathrm{nM})$. Total amounts of DNA were adjusted with pcDNA3.1. Relative Enhancer I//I activity was measured 48 hours post transfection. Transfection efficiency was normalised by $\beta$-gal assay. ${ }^{* *} \mathrm{p}<0.001 ;{ }^{* *} \mathrm{p}=0.001$; siU, siRNA control. Right panel, effect of endogenous TRIM22 knockdown on Enhancer I/II activity. HepG2 cells were transfected with pEnhl-II-Luc, pcDNA3.1, pß-gal and siRNAs, and treated with IFN $\gamma\left(500\right.$ units $/ \mathrm{mL}$ ) for 24 hours before luciferase analysis. ${ }^{*}{ }^{*} \mathrm{p}=0.001 ;{ }^{*} \mathrm{p}=0.004$ (right panel). (C) Effect of TRIM22 knockdown on viral transcription and protein expression. Cells were transfected with the indicated plasmids and siRNAs $(20 \mathrm{nM})$, and transcription (left panel) and expression of viral proteins (right panel) were analysed 72 hours post transfection by northern blotting, western blotting and ELISA. siGL3 was used as a negative control. Relative HBV RNA transcription was calculated from data obtained from three independent experiments and quantified using a phosphorimager. Secreted HBV surface antigen was analysed in culture medium. ${ }^{* *}{ }^{*} p<0.001 ;{ }^{*} p=0.006$. (D) Effect of endogenous TRIM22 knockdown on IFN $\gamma$-mediated anti-HBV activity. HepG2 cells were transfected with HBV1.2 and indicated siRNAs $(20 \mathrm{nM})$, and treated with or without IFN $\gamma(500$ units/mL) for 24 hours before harvesting. At 72 hours post transfection, the level of viral transcription was analysed by northern blotting. The expression level of the core protein and TRIM22 knockdown were confirmed by western blotting (left panel). The relative levels of total and genomic RNA ( $3.5 \mathrm{~kb}$ ) were calculated using phosphorimager data from five independent experiments. ${ }^{* * \star} \mathrm{p}<0.001$ (right panels). (E-F) Effect of TRIM22 knockdown on IFN-mediated antiviral activity in HepG2-NTCP (E) and HepaRG (F) HBV infection systems. Expression levels of HBeAg/ HBsAg and TRIM22 were measured by ELISA and western blotting, respectively. The level of HBV DNA was quantified using real-time PCR. (G) Enhancement of IFN-induced anti-HBV activity by 5AzaC. Cells transfected with HBV1.2 were treated with 5AzaC $(10 \mu \mathrm{M})$ and/or IFN $(500$ units/ $\mathrm{mL})$. HBV transcription and protein expression were analysed by northern and western blotting, respectively. Relative HBV transcription was calculated from the data from three independent experiments. ${ }^{* * *} p<0.001$.

treatment, whereas the expression of TRIM22 increased (figure 5G).

Consequently, our data indicate that TRIM22 mediates IFN $\gamma$-induced anti-HBV activity and that HBV may escape this activity through epigenetic suppression of TRIM22 by HBx.
HBV suppresses IFN-induced TRIM22 expression through HBx in a mouse model, primary human hepatocytes, and human liver tissues

To check the biological relevance of our findings in vivo, we used an acute HBV infection model described previously. ${ }^{9}$ 
E
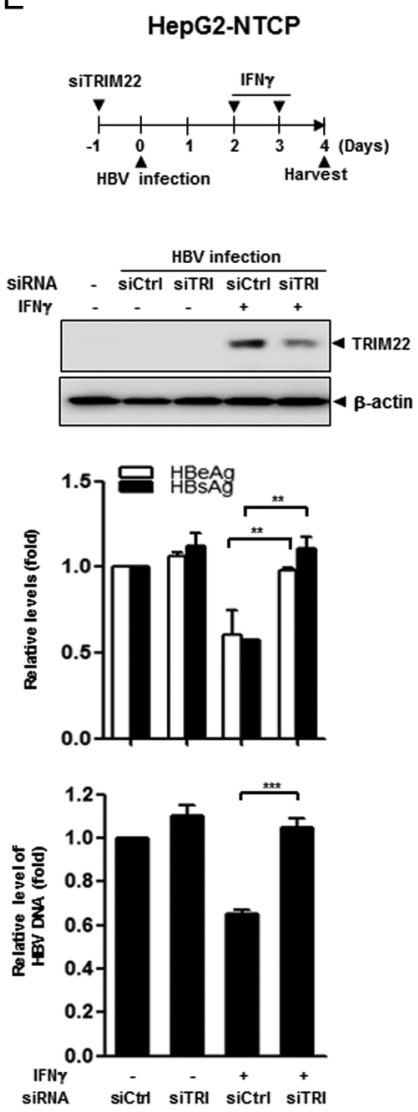

HepaRG
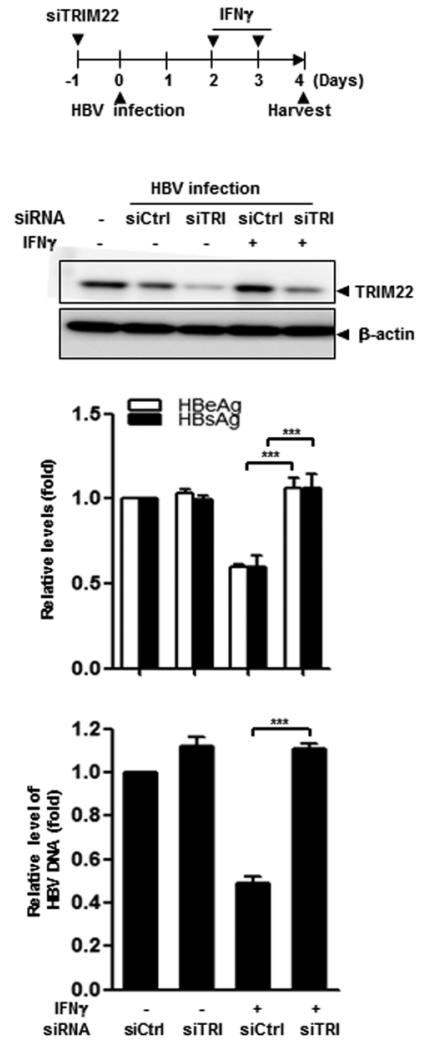

G
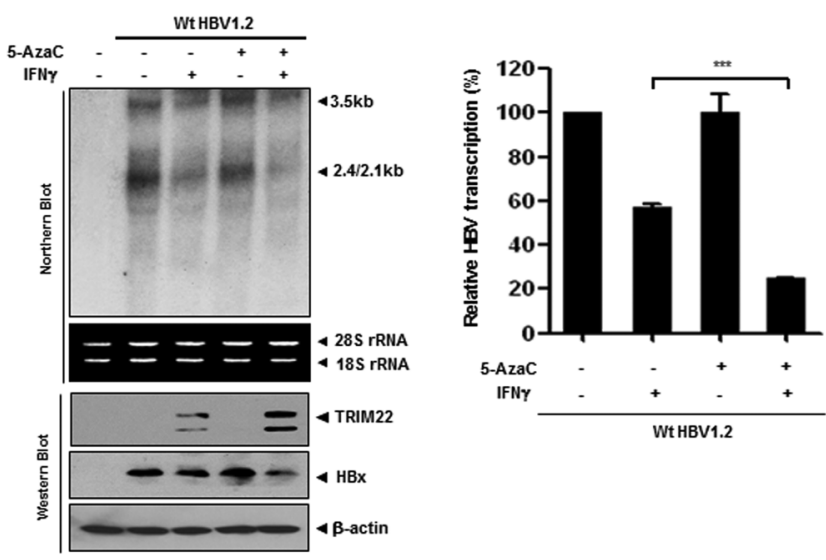

Because mice lack theTRIM22 gene, we introduced TRIM22 promoter clones (pTP5-Luc or pTP6-Luc) with indicated plasmids (pcDNA, HBx-HA, HBV1.2(X-) or WTHBV1.2) using hydrodynamic injection to access the role of $\mathrm{HBx}$ in IFN-inducible TRIM22 expression (figure 6A).

The mouse model of acute HBV infection was validated by observing HBV replication and viral protein expression (HBx-HA and HBsAg) in mouse hepatocytes (see online supplementary figure S7). Since HBx is critical for HBV replication, ${ }^{34}$ the replication of HBV1.2(X-) in the liver was marginal compared with that of WT HBV1.2 (see online supplementary figure S7A). However, there was no significant difference in the expression levels of surface proteins (see online supplementary figure $57 \mathrm{~B}$ ). Introduction of foreign DNA strongly induced type I and type II IFNs (IFN $\alpha$, IFN $\beta$ and IFN $\gamma$ ) (figure 6B). Luciferase assays showed that only the TRIM22 promoter containing the $5^{\prime}$-UTR $(+1$ to +211$)$ (pTP6-Luc) was strongly activated in response to IFNs. TRIM22 induction in mouse liver tissues was dramatically suppressed by $\mathrm{HBx}$ (figure 6C). Furthermore, replication-competent WT HBV1.2, which is capable of expressing genome-driven $\mathrm{HBx}$, strongly reduced TRIM22 promoter activity in vivo compared with the effect of HBV1.2(X-) (figure 6D). The latter observation suggests that suppression of TRIM22 by $\mathrm{HBx}$ occurs during the natural course of $\mathrm{HBV}$ infection. In addition, we tested epigenetic regulation of $\mathrm{HBx}$ in vivo by examining whether $5 \mathrm{AzaC}$ can abolish the repression of the TRIM22 promoter by HBx (figure 6E). Tail vein injection of $5 \mathrm{AzaC}$ strongly restored the activity of the pTP6 promoter (figure 6F), indicating that the $5^{\prime}$-UTR of TRIM22 is also critical and responsible for HBx-mediated suppression of TRIM22 expression in vivo.
We performed similar experiments in primary human hepatocytes (PHHs) to check the physiological relevance of our findings. To determine the infection efficiency in $\mathrm{PHHs}$, the cells were infected with various amounts of HBV (100-2000 viral genome equivalents per cell) and were analysed 7 days after infection by measuring the levels of secreted HBeAg and HBsAg. ${ }^{35}$ The percentage of HBV infection was approximately $90 \%$ (figure 7A). A slightly lower infection rate $(\sim 70 \%)$ was observed in HepaRG cells (see online supplementary figure $\mathrm{S} 8$ ).

To test whether HBV infection suppresses the expression of TRIM22 through epigenetic regulation in $\mathrm{PHHs}$, we examined the expression level of TRIM22 using real-time PCR and western blot analysis after $5 \mathrm{AzaC}$ treatment. Notably, PHHs produced a basal level of TRIM22, which was marginal in HepG2 cells (figure 2). HBV infection strongly repressed the expression of IFN $\gamma$-induced TRIM22, but not that of other ISGs such as STAT1, p-STAT1, PKR, ISG15 or IRF1 in PHHs (figure 7B). Both mRNA and protein levels of TRIM22 were dramatically restored by $5 \mathrm{AzaC}$ treatment, indicating that the suppression of TRIM22 by HBV is mediated by epigenetic regulation in PHHs (figure 7C). To assess the clinical implications of our finding, we analysed HBV-positive and HBV-negative human liver tissues by real-time PCR to determine the TRIM22 levels (baseline characteristics of included patients are provided in online supplementary table S3). The TRIM22 mRNA level was significantly lower in HBV-positive tissues (figure 7D). The level of the TRIM22 protein was also significantly decreased in HBV-positive tissues (figure 7E). The level of the TRIM22 transcript was inversely correlated with that of HBx mRNA in HBV-positive liver tissues (figure 7F) (Pearson $\mathrm{r}=-0.6551, \mathrm{p}<0.05$ ). 


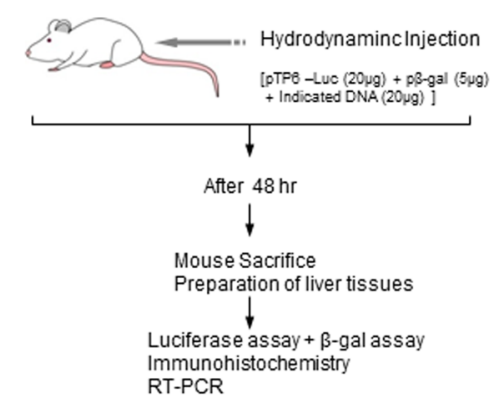

C

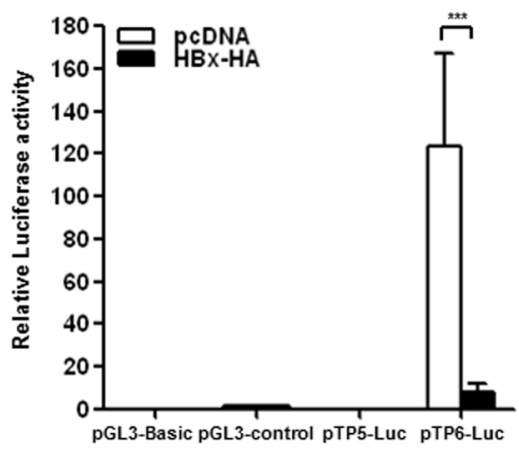

E

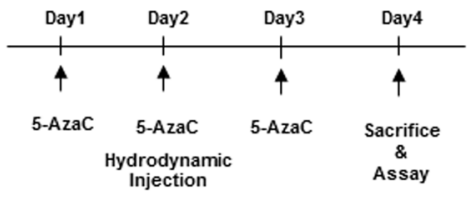

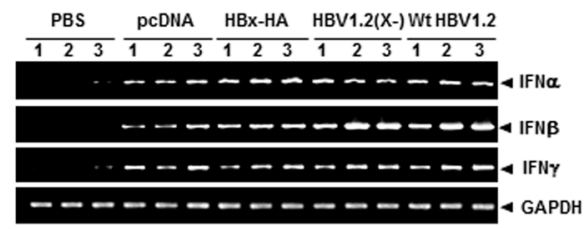

Figure 6 HBV suppresses interferons (IFNs)-inducible TRIM22 via HBV X protein (HBx) in a mouse model. (A) The experimental scheme. (B) Induction of IFNs in mouse liver by hydrodynamic injection of plasmids. At 48 hours after hydrodynamic injection, total RNA was extracted from three mouse liver tissues. The mRNA levels of mouse IFNs (mIFN $\alpha$, mIFN $\beta$ and mIFN $\gamma$ ) were analysed by semiquantitative RT-PCR. (C) Effect of HBx on TRIM22 promoter activity in liver. Reporter plasmids were hydrodynamically injected into mouse liver, and luciferase activity was analysed in liver lysates. Relative luciferase activity was measured in nine independent experiments and normalised using $\beta$-gal assay. ${ }^{* * *} p<0.001$. (D) Effect of genome-driven HBx on TP6 activity in mouse liver ( $n=5$ per group). ${ }^{* *} p<0.001$. (E) Experimental scheme of $5 \mathrm{AzaC}(5 \mathrm{mg} / \mathrm{kg})$ injection into the mouse tail vein. (F) Effect of $5 \mathrm{AzaC}$ on HBx-mediated suppression of TRIM22 promoter activity. Three mice per group were used for luciferase analysis. ${ }^{* * *} \mathrm{p}<0.001 ; \mathrm{NS}$, not significant.

\section{DISCUSSION}

The well-developed evasion strategies of some viruses allow them to survive despite the IFN response. Viruses such as $\mathrm{HIV}$ and influenza virus possess various mechanisms to outmanoeuvre the IFN response. ${ }^{36} 37$ Hepatitis viruses including $\mathrm{HCV}$, HDV and HEV also impair the IFN response. ${ }^{38-40}$ Since pegylated-IFN treatment for chronic HBV shows a poor response, ${ }^{18} 19$ it has been speculated that $\mathrm{HBV}$ has an IFN evasion mechanism, but its strategy to circumvent the IFN response is not fully understood.

In this study, we demonstrated the ability of HBV to counteract the induction of TRIM22, a mediator of the IFN-induced antiviral response. Proteome-wide analysis identified the ISG candidates suppressed by $\mathrm{HBx}$ during viral escape (figure 1). We found that HBV significantly desensitises the IFN-mediated induction of TRIM22 through methylation of a single $\mathrm{CpG}$ site in the $5^{\prime}$-UTR in vitro and in vivo. This possibly leads to HBV escape from IFN and antiviral activity of TRIM22 (figure 7G). Because HBx suppresses TRIM22 induced by either IFN $\alpha$ or IFN $\gamma$ (figure 2C, D), it is likely that $\mathrm{HBx}$ suppresses the antiviral responses induced by both type I and type II IFNs simultaneously. These results also suggest why the response to treatment with pegylated-IFN $\alpha$ is not satisfactory. TRIM22 specifically suppressed viral enhancer and core promoter activity (figure 5B), ${ }^{30}$ and TRIM22 knockdown substantially restored viral RNAs inhibited by IFN $\gamma$ (figure 5D-F). These results suggest that the therapeutic efficacy of IFNs could be improved by blocking TRIM22 suppression by $\mathrm{HBx}$ (figure 5G).

A number of studies have shown that epigenetic modulation of host genes is involved in viral evasion from host immune responses. ${ }^{41}$ We and others have reported that $\mathrm{HBx}$ induces epigenetic changes in host genomes. ${ }^{89}$ Here, we found a host epigenetic target involved in HBV escape from host immune responses. Epigenetic control is also involved in basal induction 
A

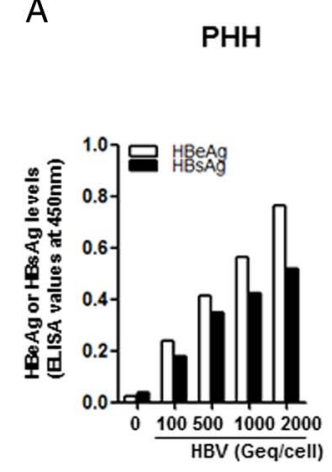

PHH
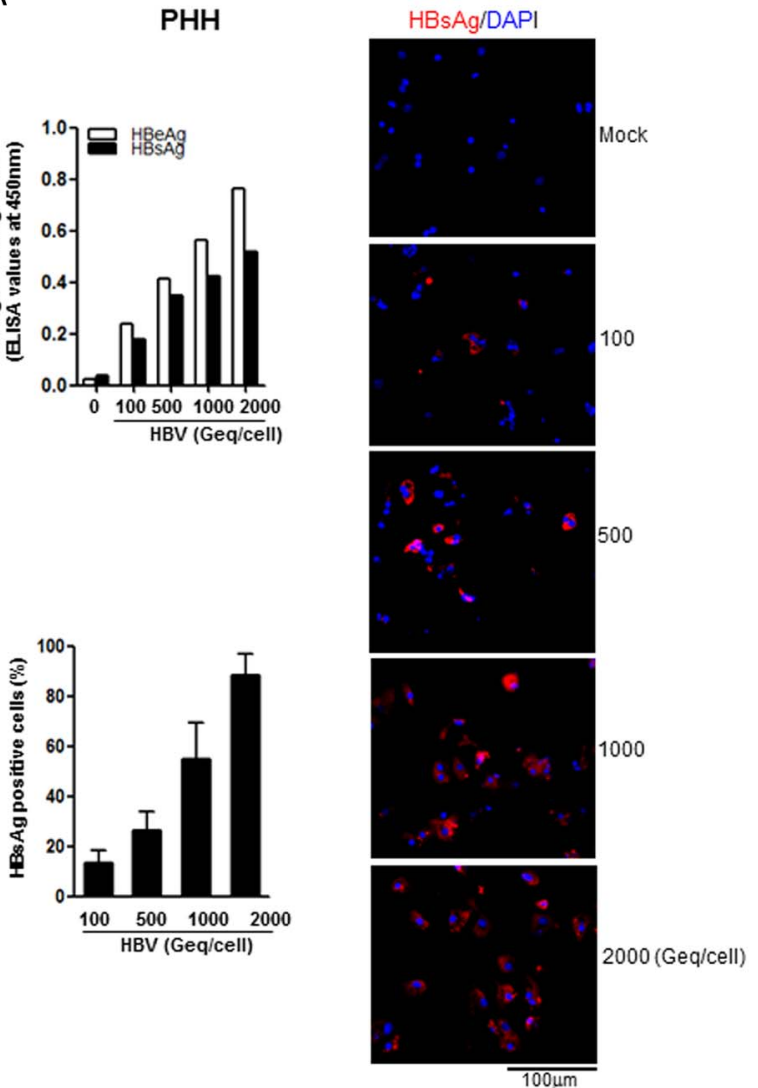

B
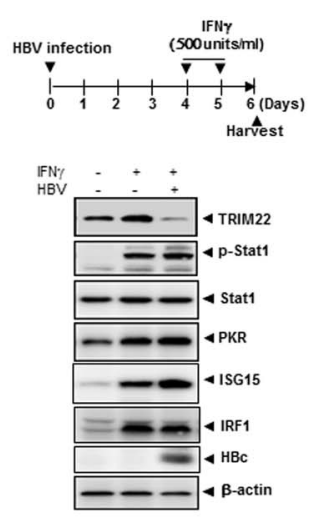

D

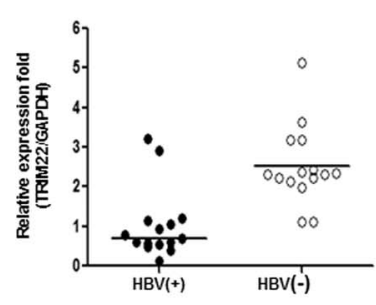

C
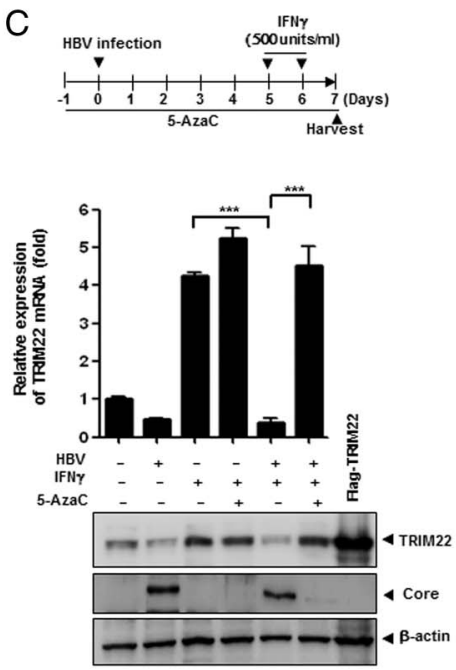

E

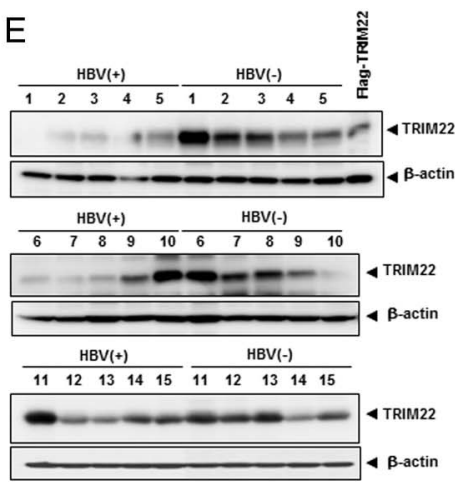

F

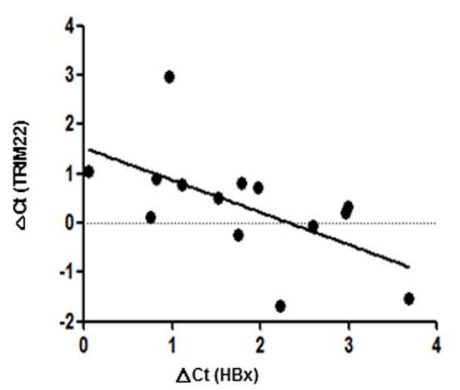

G

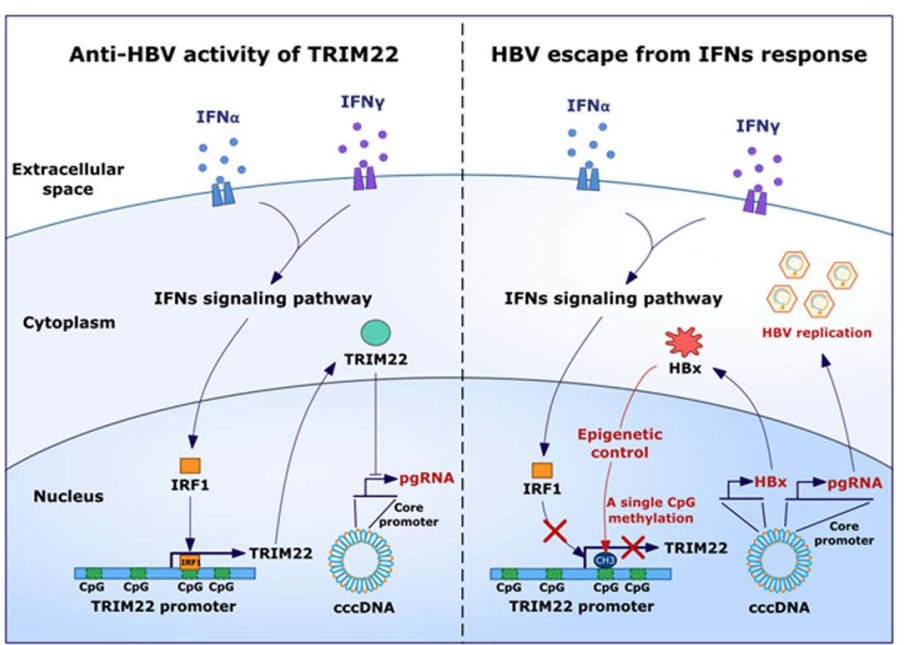

Figure 7 HBV suppresses TRIM22 induced by interferons (IFNs) in primary human hepatocytes (PHHs) and human liver tissues. (A) Efficiency of HBV infection in PHHs. The level of secreted HBeAg and HBsAg were measured by ELISA at 7 days post infection. HBsAg-positive cells (\%) were quantitated using the confocal images. Data are mean \pm SD of at least five regions. (B) Effect of HBV infection on expression levels of TRIM22 and other IFN-stimulated genes (ISGs) in PHHs. At 6 days post infection, the indicated proteins were detected by western blotting. (C) Effect of $5 \mathrm{AzaC}$ on HBV-mediated suppression of TRIM22. At 7 days post infection, the relative expression level of TRIM22 was measured by real-time PCR and the expression of TRIM22 was determined by western blotting. Data are mean \pm SD from at least three independent experiments. ${ }^{* * *} p=0.001$. (D) TRIM22 expression levels in HBV-positive and HBV-negative liver tissues were evaluated by real-time PCR. (E) The level of the TRIM22 protein in liver tissues was determined by western blotting. (F) TRIM22 and HBV X protein (HBX) expression levels in HBV-positive tissues were measured by real-time PCR. Respective delta Ct values were subjected to Pearson correlation analysis. (G) A schematic model illustrating the epigenetic regulation of TRIM22 by HBV for viral evasion from IFN-mediated antiviral activity. IRF1, IFN regulatory factor-1.

of TRIM22 by IFNs because the expression level of IFN $\gamma$-induced TRIM22 was increased by $5 \mathrm{AzaC}$ treatment (figure $3 \mathrm{~F}$, left panel, lane 5 vs 7). Importantly, the epigenetic modulation of TRIM22 was mediated by methylation of a single CpG in the $5^{\prime}$-UTR, not CpG islands in the promoter. The control of gene expression by a single $\mathrm{CpG}$ is not common; 
however, such regulation has been discovered in both host cells and viruses, ${ }^{42}{ }^{43}$ implying that methylation at a specific single $\mathrm{CpG}$ could be sufficient for adaptation to environmental change.

The level of HBV replication in IRF1 knockout mice was higher than that in wild type, ${ }^{12}$ suggesting that some factors regulated by IFN-mediated IRF1 may be involved in HBV inhibition. We identified TRIM22 as an IRF1-regulated antiviral protein and established that its $5^{\prime}$-UTR is essential for both IFN-mediated induction and $\mathrm{HBx}$-mediated suppression of TRIM22 (figure 3A-C). Although HBx induced methylation at four $\mathrm{CpG}$ sites (figure 3D), only methylation at a specific single CpG (+71) decreased IRF1 binding to the $5^{\prime}$-UTR of TRIM22 (figure 4) and subsequent IFN-induced TRIM22 expression. However, the expression of IRF1 was not changed by HBx (see online supplementary figure S4). Demethylation of the third CpG upon $5 \mathrm{AzaC}$ treatment restored IFN $\gamma$-mediated TRIM22 expression in HBx-expressing cells (figure 3E, F). Our study suggests that single-nucleotide polymorphism at this critical CpG site in TRIM22 might cause different clinical outcomes in individual patients infected with HBV. A clinical study might be warranted to evaluate the association between the level of TRIM22 and virological response to IFN.

Although HBV mainly infects hepatocytes, it has also been reported to infect immune cells such as human peripheral blood lymphocytes (PBMCs) including $\mathrm{T}$ lymphocytes. ${ }^{44} 45$ Coinfection with HIV and HBV has a worse prognosis in patients than infection with $\mathrm{HBV}$ alone. ${ }^{46}$ It is well known that TRIM22 has potent anti-HIV activity, and the level of TRIM22 in immune cells is higher than that in other cells. ${ }^{25}$ Therefore, we assume that downregulation of TRIM22 by HBx in PBMCs could also lead to HIV evasion from IFN-mediated immune responses in coinfected patients. Indeed, this assumption is supported by previous reports of large amounts of HBx detected in PBMCs ${ }^{44}{ }^{45}$ and by the ability of HBx to induce HIV replication and transcription and thereby contribute to a faster progression to AIDS. ${ }^{47}$

In the case of HBV, the evasion strategies against the IFN response through HBV polymerase and $\mathrm{HBx}$ have been observed in hepatocytes and mouse models. HBV polymerase and $\mathrm{HBx}$ can counteract pattern recognition receptor signalling through binding to DDX3 and IPS-1, and thereby downregulate IFN production in hepatocytes. ${ }^{22}{ }^{23}$ In addition, HBV effectively suppresses IFN signalling by impairing STAT activation through inhibition of STAT nuclear translocation. ${ }^{21}$ Since TRIM22 is induced by both type I and type II IFNs, the suppression of TRIM22 by HBx could have a role in HBV escape from a broad range of host IFNs involved in the defence system. It is worth noting that the steady-state protein level of TRIM22 is higher in HepaRG and PHHs compared with HepG2 cells (figures 5E-F and 7B, C). This high level of TRIM22 in PHHs may serve as a restriction factor for HBV spread at early stage of infection. Although the details of the mechanisms of HBV evasion from the IFN-mediated antiviral response are still largely unclear, our finding will help to understand how HBV acts like a 'stealth' virus. 48

The liver contains non-parenchymal cells including Kupffer cells, sinusoidal endothelial cells and lymphocytes, and is an organ with predominant innate immunity. ${ }^{49}$ Thus, cytokines (including IFNs) secreted from non-parenchymal cells may contribute to the paracrine anti-HBV response in hepatocytes, although HBV can block the IFN response in hepatocytes. Interestingly, it was recently reported that IFN $\alpha$-induced anti-HBV activity is transferred by cell-to-cell transmission via exosomes from non-parenchymal cells. ${ }^{50}$ Accordingly, the strategy of HBV to directly reduce the expression of ISGs such as TRIM22 and therefore to downregulate the IFN response seems to be effective for HBV survival, because HBV cannot infect and regulate non-parenchymal cells effectively.

It has been reported that the induction of ISGs is limited and not detectable in mouse and chimpanzee during the initial phase of HBV infection, unlike infections with other viruses such as $\mathrm{HCV}^{48}$ Nevertheless, the detailed mechanism of the direct inhibition of IFN-induced antiviral proteins by HBV has not yet been clearly elucidated. Here, we found that TRIM22 induction by IFNs is downregulated by $\mathrm{HBx}$ via epigenetic control of a single CpG methylation site in the $5^{\prime}$-UTR of TRIM22. Functional analysis of other proteins from Cluster C (figure 1) would also yield information helpful for understanding HBV evasion from the IFN response.

In conclusion, we showed that epigenetic regulation of TRIM22 by $\mathrm{HBx}$ is involved in HBV evasion from the IFN-mediated antiviral response. Our findings will extend the understanding of the pathophysiology of HBV infection and provide a potential way to improve the therapeutic effect of IFNs for HBV clearance.

\section{EXPERIMENTAL PROCEDURES}

Materials and methods are described in online supplementary information.

\section{Author affiliations}

1Department of Pharmacology, Center for Cancer Research and Diagnostic Medicine, IBST, School of Medicine, Konkuk University, Seoul, Korea

${ }^{2}$ Department of Applied Chemistry, Kyung Hee University, Yongin, Gyeonggi, Korea

${ }^{3}$ Department of Anatomy, School of Medicine, Konkuk University, Seoul, Korea

${ }^{4}$ Department of Surgery, Uijeongbu St Mary's Hospital, College of Medicine, The Catholic University of Korea, Uijeongbu, Korea

${ }^{5}$ Department of Internal Medicine, Uijeongbu St Mary's Hospital, College of

Medicine, The Catholic University of Korea, Uijeongbu, Korea

${ }^{6}$ Department of Biochemistry, School of Medicine, Konkuk University, Seoul, Korea

${ }^{7}$ Department of Internal Medicine and Liver Research Institute, Seoul National

University College of Medicine, Seoul, Korea

${ }^{8}$ Department of Surgery, Seoul National University College of Medicine, Seoul, Korea

${ }^{9}$ Department of Biotechnology, College of Life Science and Biotechnology, Yonsei University, Seoul, Korea

${ }^{10} \mathrm{KU}$ Open Innovation Center, Konkuk University, Seoul, Korea

${ }^{11}$ Research Institute of Medical Sciences, Konkuk University, Seoul, Korea

Contributors Study conception and design: K-HL, E-SP and K-HK. Acquisition of data: K-HL, E-SP, KCC, YKP, DHK, SHA, SHP, HSK, ARL, SP, HS and JW. Analysis and interpretation of data: K-HL, E-SP, KPK, JSY, BLS and KHK. Material support: K-HK, CWK, J-HL, N-JY, K-WL and K-SS. Obtained funding: K-HK and E-SP. Drafted the manuscript: K-HL, E-SP and K-HK.

Funding This study was supported by the National Research Foundation (NRF) grant funded by the Korean government (No. 2013R1A2A2A01068194, No. 2014M3A9A8064633, NRF-2016R1A5A2012284 and 2016R1A2B4007531). This research was also supported by a grant of the Korea Health Technology R\&D Project through the Korea Health Industry Development Institute, funded by the Ministry of Health and Welfare, Republic of Korea (grant number: HI14C-1529-020014).

Competing interests None declared.

\section{Patient consent Obtained.}

Provenance and peer review Not commissioned; externally peer reviewed.

\section{REFERENCES}

1 García-Sastre A, Biron CA. Type 1 interferons and the virus-host relationship: a lesson in détente. Science 2006;312:879-82.

2 Takaoka A, Yanai H. Interferon signalling network in innate defence. Cell Microbiol 2006;8:907-22.

3 Sadler AJ, Williams BR. Interferon-inducible antiviral effectors. Nat Rev Immunol 2008:8:559-68

4 Ganem D, Prince AM. Hepatitis B virus infection - natural history and clinical consequences. N Engl J Med 2004;350:1118-29. 
5 Hirsch RC, Lavine JE, Chang LJ, et al. Polymerase gene products of hepatitis B viruses are required for genomic RNA packaging as well as for reverse transcription. Nature 1990;344:552-5.

6 Seeger C, Mason WS. Hepatitis B virus biology. Microbiol Mol Biol Rev 2000;64:51-68.

7 Bouchard MJ, Schneider RJ. The enigmatic $X$ gene of hepatitis B virus. J Virol 2004:78:12725-34

8 Park IY, Sohn BH, Yu E, et al. Aberrant epigenetic modifications in hepatocarcinogenesis induced by hepatitis B virus X protein. Gastroenterology 2007;132:1476-94

9 Park ES, Park YK, Shin CY, et al. Hepatitis B virus inhibits liver regeneration via epigenetic regulation of urokinase-type plasminogen activator. Hepatology 2013:58:762-76.

10 Belloni L, Allweiss L, Guerrieri F, et al. IFN- $\alpha$ inhibits HBV transcription and replication in cell culture and in humanized mice by targeting the epigenetic regulation of the nuclear cccDNA minichromosome. J Clin Invest 2012;122:529-37.

11 Li J, Lin S, Chen Q, et al. Inhibition of hepatitis B virus replication by MyD88 involves accelerated degradation of pregenomic RNA and nuclear retention of pre-S/ S RNAs. J Virol 2010;84:6387-99.

12 Guidotti LG, Morris A, Mendez H, et al. Interferon-regulated pathways that control hepatitis B virus replication in transgenic mice. J Virol 2002;76:2617-21.

13 Wieland SF, Guidotti LG, Chisari FV. Intrahepatic induction of alpha/beta interferon eliminates viral RNA-containing capsids in hepatitis B virus transgenic mice. J Virol 2000;74:4165-73.

14 Xu C, Guo H, Pan XB, et al. Interferons accelerate decay of replication-competent nucleocapsids of hepatitis B virus. J Virol 2010;84:9332-40.

15 Li N, Zhang L, Chen L, et al. MxA inhibits hepatitis B virus replication by interaction with hepatitis $B$ core antigen. Hepatology 2012;56:803-11.

16 Turelli $P$, Mangeat $B$, Jost $S$, et al. Inhibition of hepatitis $B$ virus replication by APOBEC3G. Science 2004;303:1829.

17 Lok AS. Personalized treatment of hepatitis B. Clin Mol Hepatol 2015;21:1-6.

18 Brook MG, McDonald JA, Karayiannis $P$, et al. Randomised controlled trial of interferon alfa 2A (rbe) (Roferon-A) for the treatment of chronic hepatitis $B$ virus (HBV) infection: factors that influence response. Gut 1989:30:1116-22.

19 Lau GK, Piratvisuth T, Luo KX, et al. Peginterferon alfa-2a, lamivudine, and the combination for HBeAg-positive chronic hepatitis B. N Engl J Med 2005;352:2682-95.

20 Hiraga N, Imamura M, Hatakeyama T, et al. Absence of viral interference and different susceptibility to interferon between hepatitis $B$ virus and hepatitis $C$ virus in human hepatocyte chimeric mice. J Hepatol 2009;51:1046-54.

21 Chen J, Wu M, Zhang $X$, et al. Hepatitis B virus polymerase impairs interferon- $\alpha$-induced STAT activation through inhibition of importin- $\alpha 5$ and protein kinase C- $\delta$. Hepatology 2013;57:470-82.

22 Kumar M, Jung SY, Hodgson AJ, et al. Hepatitis B virus regulatory HBx protein binds to adaptor protein IPS-1 and inhibits the activation of beta interferon. $J$ Virol 2011:85:987-95.

23 Wang $H$, Ryu WS. Hepatitis B virus polymerase blocks pattern recognition receptor signaling via interaction with DDX3: implications for immune evasion. PLoS Pathog 2010;6:e1000986.

24 Ozato $\mathrm{K}$, Shin DM, Chang TH, et al. TRIM family proteins and their emerging roles in innate immunity. Nat Rev Immunol 2008:8:849-60.

25 Hattlmann CJ, Kelly JN, Barr SD. TRIM22: a diverse and dynamic antiviral protein. Mol Biol Int 2012;2012:153415.

26 Barr SD, Smiley JR, Bushman FD. The interferon response inhibits HIV particle production by induction of TRIM22. PLoS Pathog 2008;4:e1000007.

27 Tissot C, Mechti N. Molecular cloning of a new interferon-induced factor that represses human immunodeficiency virus type 1 long terminal repeat expression. J Biol Chem 1995;270:14891-8.

28 Di Pietro A, Kajaste-Rudnitski A, Oteiza A, et al. TRIM22 inhibits influenza A virus infection by targeting the viral nucleoprotein for degradation. $J$ Virol 2013:87:4523-33.
29 Eldin P, Papon L, Oteiza A, et al. TRIM22 E3 ubiquitin ligase activity is required to mediate antiviral activity against encephalomyocarditis virus. J Gen Virol 2009;90:536-45

30 Gao B, Duan Z, Xu W, et al. Tripartite motif-containing 22 inhibits the activity of hepatitis $B$ virus core promoter, which is dependent on nuclear-located RING domain. Hepatology 2009;50:424-33.

31 Wieland S, Thimme R, Purcell RH, et al. Genomic analysis of the host response to hepatitis B virus infection. Proc Natl Acad Sci USA 2004;101: 6669-74

32 Obad S, Brunnström H, Vallon-Christersson J, et al. Staf50 is a novel p53 target gene conferring reduced clonogenic growth of leukemic U-937 cells. Oncogene 2004:23:4050-9.

33 Gao B, Wang Y, Xu W, et al. A $5^{\prime}$ extended IFN-stimulating response element is crucial for IFN-gamma-induced tripartite motif 22 expression via interaction with IFN regulatory factor-1. J Immunol 2010;185:2314-23.

34 Bouchard MJ, Wang LH, Schneider RJ. Calcium signaling by HBx protein in hepatitis B virus DNA replication. Science 2001:294:2376-8.

35 Park YK, Park ES, Kim DH, et al. Cleaved c-FLIP mediates the antiviral effect of TNF- $\alpha$ against hepatitis $B$ virus by dysregulating hepatocyte nuclear factors. J Hepatol 2016;64:268-77

36 Marsili G, Remoli AL, Sgarbanti M, et al. HIV-1, interferon and the interferon regulatory factor system: an interplay between induction, antiviral responses and viral evasion. Cytokine Growth Factor Rev 2012;23:255-70.

37 Schmolke M, García-Sastre A. Evasion of innate and adaptive immune responses by influenza A virus. Cell Microbiol 2010;12:873-80.

38 Dong C, Zafrullah M, Mixson-Hayden T, et al. Suppression of interferon- $\alpha$ signaling by hepatitis E virus. Hepatology 2012;55:1324-32.

39 Gale MJ. Foy EM. Evasion of intracellular host defence by hepatitis C virus. Nature 2005;436:939-45.

40 Pugnale P, Pazienza V, Guilloux K, et al. Hepatitis delta virus inhibits alpha interferon signaling. Hepatology 2009;49:398-406.

41 Adhya D, Basu A. Epigenetic modulation of host: new insights into immune evasion by viruses. J Biosci 2010;35:647-63.

42 Robertson KD, Hayward SD, Ling PD, et al. Transcriptional activation of the Epstein-Barr virus latency $C$ promoter after 5-azacytidine treatment: evidence that demethylation at a single CpG site is crucial. Mol Cell Biol 1995;15: 6150-9.

43 Sohn BH, Park IY, Lee JJ. Functional switching of TGF-beta1 signaling in liver cance via epigenetic modulation of a single CpG site in TTP promoter. Gastroenterology 2010;138:1898-908.

44 Jung MC, Stemler M, Weimer T, et al. Immune response of peripheral blood mononuclear cells to HBx-antigen of hepatitis B virus. Hepatology 1991;13: 637-43.

45 Stoll-Becker S, Repp R, Glebe D, et al. Transcription of hepatitis B virus in peripheral blood mononuclear cells from persistently infected patients. J Virol 1997:71:5399-407.

46 Chun HM, Roediger MP, Hullsiek KH, et al. Hepatitis B virus coinfection negatively impacts HIV outcomes in HIV seroconverters. J Infect Dis 2012;205: 185-93.

47 Gómez-Gonzalo M, Carretero M, Rullas J, et al. The hepatitis B virus X protein induces HIV-1 replication and transcription in synergy with T-cell activation signals: functional roles of NF-kappaB/NF-AT and SP1-binding sites in the HIV-1 long terminal repeat promoter. J Biol Chem 2001;276:35435-43.

48 Wieland SF, Chisari FV. Stealth and cunning: hepatitis B and hepatitis C viruses J Virol 2005:79:9369-80.

49 Gao B, Jeong WI, Tian Z. Liver: An organ with predominant innate immunity. Hepatology 2008:47:729-36.

50 Li J, Liu K, Liu Y, et al. Exosomes mediate the cell-to-cell transmission of IFN- $\alpha$-induced antiviral activity. Nat Immunol 2013;14:793-803. 